\title{
Synthesis, Characterization, and Antibacterial Activities of Novel Sulfonamides Derived through Condensation of Amino Group Containing Drugs, Amino Acids, and Their Analogs
}

\author{
Muhammad Abdul Qadir, ${ }^{1}$ Mahmood Ahmed, ${ }^{1}$ and Muhammad Iqbal ${ }^{2}$ \\ ${ }^{1}$ Institute of Chemistry, University of Punjab, Lahore 54590, Pakistan \\ ${ }^{2}$ Department of Chemistry, Minhaj University, Lahore, Pakistan \\ Correspondence should be addressed to Mahmood Ahmed; mahmoodresearchscholar@gmail.com
}

Received 16 December 2014; Accepted 9 February 2015

Academic Editor: Ajit S. Narang

Copyright (C) 2015 Muhammad Abdul Qadir et al. This is an open access article distributed under the Creative Commons Attribution License, which permits unrestricted use, distribution, and reproduction in any medium, provided the original work is properly cited.

\begin{abstract}
Novel sulfonamides were developed and structures of the new products were confirmed by elemental and spectral analysis (FT-IR, ESI-MS, ${ }^{1} \mathrm{HNMR}$, and $\left.{ }^{13} \mathrm{CNMR}\right)$. In vitro, developed compounds were screened for their antibacterial activities against medically important gram (+) and gram (-) bacterial strains, namely, S. aureus, B. subtilis, E. coli, and K. pneumoniae. The antibacterial activities have been determined by measuring MIC values $(\mu \mathrm{g} / \mathrm{mL})$ and zone of inhibitions $(\mathrm{mm})$. Among the tested compounds, it was found that compounds $5 \mathrm{a}$ and $9 \mathrm{a}$ have most potent activity against $E$. coli with zone of inhibition: $31 \pm 0.12 \mathrm{~mm}$ (MIC: $7.81 \mu \mathrm{g} / \mathrm{mL}$ ) and $30 \pm 0.12 \mathrm{~mm}$ (MIC: $7.81 \mu \mathrm{g} / \mathrm{mL}$ ), respectively, nearly as active as ciprofloxacin (zone of inhibition: $32 \pm 0.12 \mathrm{~mm}$ ). In contrast, all the compounds were totally inactive against the gram (+) B. subtilis.
\end{abstract}

\section{Introduction}

Sulfa drugs containing sulfonamide functional group which have extensive biological activities revolutionised the field of medical sciences [1]. Folic acid, an important chemical for synthesis of bacterial DNA and RNA, is inhibited by sulfonamides; production of new DNA and RNA is decreased by the deficiency of tetrahydrofolate which ultimately decayed the bacteria. Microorganism's normal growth is inhibited due to mistaken attempt by bacteria to convert sulfonamide instead of $p$-amino benzoic acid for synthesis of folic acid. Due to such activities sulfonamides are also efficiently used in agriculture field for antibacterial activities $[2,3]$. Inhibition of carbonic anhydrases has been done by the drugs containing sulfonamide functional group and these carbonic anhydrase inhibitors are also reported as potential anticancer, antiglaucoma (as substituted heterocyclic and aromatic sulfonamides), diuretics, and antiobesity agents [4]. Therapeutically sulfonamides are being widely used in human (especially where other antibiotics are nontolerable to patients) and veterinary practice [5]. For agricultural purposes many derivatives of sulfonamide have been reported due to their antifungal [6-8] and herbicidal [9] properties. The newer antibacterial drugs with different mode of action and mechanism have become an emerging demand to overcome existing drugs resistant pathogens. As the pathogenic organisms (bacteria, fungi, and mold) are exposed or treated medically with routine antibiotic drug molecules, they become considerably resistant with emergence of new species as per mutation, conjugation, transduction, or transformation. The synthesis of new sulfonamides has got more attention of researchers for their previous success in the field of pharmaceutical sciences and medicinal chemistry. In recent studies, ten new sulfonamides have been synthesized and characterized by the reaction of p-toluene sulphonyl chloride with essential amino acids (histidine and tryptophan) and amino group containing drugs such as levetiracetam (anticonvulsant), famotidine (antiulcer), celecoxib (NSAID), ribavirin (antiviral), tranexamic acid (antifibrinolytic), furosemide (diuretic), aspartame (nonsaccharide sweetener), and nicotinamide (vitamin B), 
respectively. Antibacterial activities of synthesized compounds were also evaluated against both gram $(+)$ and gram (-) bacteria.

\section{Experimental}

2.1. Chemistry. Chemicals used in present work were of analytical grade obtained from E-Merck (Germany) and $\mathrm{BDH}(\mathrm{UK})$ without further purification to synthesize desired compounds; grade 1 quality water $(0.01 \mu \mathrm{S} / \mathrm{cm})$ [10] was prepared in our own laboratory. Alpha IR spectrometer (FTIR-ATR) and Bruker and NMR spectrometer, Bruker, were used to record the IR and ${ }^{1} \operatorname{HNMR}(500 \mathrm{MHz})$ and ${ }^{13} \mathrm{CNMR}(125 \mathrm{MHz})$ spectra, respectively. PG-T80 ${ }^{+} \mathrm{UV}$-vis spectrophotometer and Flash HT Plus elemental analyzer, Thermo Scientific, were used for $\lambda_{\max }$ and concentration of hydrogen $(\mathrm{H})$, carbon $(\mathrm{C})$, nitrogen $(\mathrm{N})$, and sulfur $(\mathrm{S})$ of respective synthesized compounds, respectively, while the melting point was measured by Gallenkamp apparatus. JMSHX-110 spectrometer with electron ionization interface was used for mass spectra. The ${ }^{1} \mathrm{HNMR}$ and ${ }^{13} \mathrm{CNMR}$ spectra of all the synthesized compounds were measured using MeOD and concentration of all the compounds was $10-20 \mathrm{mg}$ in $0.8-$ $1.0 \mathrm{~mL}$ of solvent. Purification and progress of the synthesized compounds were confirmed on precoated TLC silica plate (Merck, Germany).

2.2. Antibacterial Activity Assay. Escherichia coli ATCC 25922, Staphylococcus aureus ATCC 25923, Bacillus subtilis ATCC 6633, and Klebsiella pneumoniae ATCC 13887 were collected from Mycology Department, University of Punjab, Lahore, Pakistan, and were maintained in tryptic soy agar (TSA) medium slants at $5^{\circ} \mathrm{C}$ until use. A series of ten 2-fold dilutions were made by dissolving $10-30 \mathrm{mg}$ of each sulfonamide separately in $1 \mathrm{~mL}$ dimethyl sulphoxide (DMSO). All the dilutions were made sterile in an autoclave at $121^{\circ} \mathrm{C}$ for $30 \mathrm{~min}$ with $15 \mathrm{psi}$ pressure after filtration through $0.22 \mu \mathrm{m}$ membrane filter. The minimal inhibitory concentration (MIC) was reported as absence of no observable growth by the lowest concentration of tested compounds after twofold serial dilution. Ten individually numbered test tubes with screw capped are sterilized. Tube 1 was filled with $2 \mathrm{~mL}$ of tryptic soy broth culture media including the stock solution of synthesized compounds. $1.0 \mathrm{~mL}$ of this solution was introduced into Tube 2 and diluted with $1.0 \mathrm{~mL}$ culture media and the procedure was repeated up to Tube 10. The concentration of all the compounds used for MIC value was $2-0.0039 \mathrm{mg} / \mathrm{mL}$ obtained by twofold serial dilution technique. The tubes were incubated at $25^{\circ} \mathrm{C}$ for $72 \mathrm{hrs}$. Ciprofloxacin was used as reference (positive control to check the sensitivity of tested bacterial strains). $1-3 \times 10^{8} \mathrm{cfu} / \mathrm{mL}$ of each of gram negative E. coli and $K$. pneumoniae and gram positive $S$. aureus and $B$. subtilis were obtained after adjusting the optical density of inoculum at 0.2-0.3 and 0.3$0.4(620 \mathrm{~nm})$, respectively. All the compounds and reference solutions were applied $(50 \mu \mathrm{L})$ onto a $6 \mathrm{~mm}$ sterile filter paper disc separately and the inoculated plates were incubated at $37^{\circ} \mathrm{C}$ for $24 \mathrm{hrs}$. The zones of inhibition $(\mathrm{mm})$ were measured and the antibacterial activities were evaluated. Studies were performed in triplicate and zone of inhibition was calculated with the mean $\pm \mathrm{SD}$ values.

2.3. General Procedure for Synthesis of Sulfonamides. A simple method in aqueous media under dynamic $\mathrm{pH}$ control is adopted for synthesis of sulfonamides. Filtration after acidification is involved for isolation of products [11]. All the drugs were weighed accurately and dissolved completely by addition of distilled water by constant stirring using magnetic stirrer. The $\mathrm{pH}$ of the reaction contents was strictly monitored and maintained at 8-10 at regular intervals during the experimental reaction using $\mathrm{Na}_{2} \mathrm{CO}_{3}$ solution $(1 \mathrm{M})$. Then p-toluene sulphonyl chloride was accurately weighed and added carefully into the above solution. The reaction was carried in round bottom flask equipped with magnetic stirrer. Alkaline environment made the removal of hydrogen easier. During stirring p-toluene sulphonyl chloride initially floats on the surface and the completion of reaction was examined by the change in $\mathrm{pH}$ value due to formation of $\mathrm{HCl}$ by the consumption of p-toluene sulphonyl chloride during the reaction. On completion of the reaction $\mathrm{pH}$ was adjusted at 2-3 using $\mathrm{HCl}$ solution (2 M). The precipitates formed were filtered through Whatman Filter Paper No. 42, washed several times with distilled water and recrystallized using methanol, and finally washed with water and acetone $(9: 1)$ and dried over anhydrous $\mathrm{MgSO}_{4}$. Products formation was confirmed through TLC (methanol: water : acetone in $60: 20: 20$ ratio).

\subsection{Spectral Characterization of Sulfonamides}

2.4.1. N-[(4-Methylphenyl) sulfonyl] Nicotinamide (3a). $611.0 \mathrm{mg}(5.0 \mathrm{mmol})$ nicotinamide reacts with $954.0 \mathrm{mg} p$ toluenesulfonyl chloride $(5.0 \mathrm{mmol})$ and 3 a compound was obtained as white solid. IR $v_{\max }\left(\mathrm{cm}^{-1}\right): 3062$ (C-H $\left.\mathrm{H}_{\text {aromatic, stretching }}\right), \quad 2978 \quad\left(\mathrm{C}-\mathrm{H}_{\text {methyl, stretching }}\right), \quad 1705$ $\left(\mathrm{C}=\mathrm{O}_{\text {carbonyl }}\right), 1620\left(\mathrm{~N}-\mathrm{H}_{\text {bending }}\right), 1018\left(\mathrm{~S}=\mathrm{O}_{\text {stretching }}\right), 1126$ $\left(-\mathrm{N}-\mathrm{S}=\mathrm{O}_{\text {stretching }}\right), 1458 \quad\left(\mathrm{C}=\mathrm{C}-\mathrm{C}_{\text {aromatic, stretching }}\right), 706 \quad(\varnothing-$ $\left.\mathrm{S}_{\text {stretching }}\right) ;{ }^{1} \mathrm{HNMR}(\mathrm{MeOD}, \delta / \mathrm{ppm}): 9.11$ (s, $\left.1 \mathrm{H}, \mathrm{CH}\right), 8.98$ (d, $J=5.08 \mathrm{~Hz}, 1 \mathrm{H}, \mathrm{CH}), 8.38(\mathrm{~s}, 1 \mathrm{H}, \mathrm{NH}), 8.06(\mathrm{~d}, J=8.29 \mathrm{~Hz}$, $1 \mathrm{H}, \mathrm{CH}), 7.81(\mathrm{q}, J=7.26 \mathrm{~Hz}, 1 \mathrm{H}, \mathrm{CH}), 7.42(\mathrm{~d}, J=8.21 \mathrm{~Hz}$, $1 \mathrm{H}, \mathrm{CH}), 2.45$ (s, 3H, $\left.\mathrm{CH}_{3}\right) ;{ }^{13} \mathrm{CNMR}(\mathrm{MeOD}, \delta / \mathrm{ppm}): 164$ (C-7), 151 (C-2), 146.8 (C-6), 137 (C-4), 129 (C-17), 126 (C-18), 22 (C-19); ESI-MS: $m / z 278.48[\mathrm{M}+2]^{+}, 276.34[\mathrm{M}]^{+}$.

2.4.2. (2S)-N-[(4-Methylphenyl) sulfonyl]-2-(2-oxopyrrolidin1-yl) Butanamide (3b). $567.0 \mathrm{mg}(3.34 \mathrm{mmol})$ levetiracetam reacts with $635.0 \mathrm{mg} p$-toluenesulfonyl chloride $(3.34 \mathrm{mmol})$ and $3 \mathrm{~b}$ compound was obtained as white solid. IR $v_{\max }$ $\left(\mathrm{cm}^{-1}\right): 3363\left(\mathrm{~N}-\mathrm{H}_{\text {amine, stretching }}\right), 3063\left(\mathrm{C}-\mathrm{H}_{\text {aromatic, stretching }}\right)$, $2960\left(\mathrm{C}-\mathrm{H}_{\text {methyl, stretching }}\right), 1720 \quad\left(\mathrm{C}=\mathrm{O}_{\text {carbonyl }}\right), 1658 \quad(\mathrm{~N}-$ $\left.\mathrm{H}_{\text {bending }}\right), 1010$ ( $\left.\mathrm{S}=\mathrm{O}_{\text {stretching }}\right), 1144\left(-\mathrm{N}-\mathrm{S}=\mathrm{O}_{\text {stretching }}\right), 1504$ $\left(\mathrm{C}=\mathrm{C}-\mathrm{C}_{\text {aromatic, stretching }}\right), 675\left(\varnothing-\mathrm{S}_{\text {stretching }}\right) ;{ }^{1} \mathrm{HNMR}(\mathrm{MeOD}$, $\delta / \mathrm{ppm}$ ): 9.68 (br s, $1 \mathrm{H}, \mathrm{NH}), 7.61$ (d, $J=8.24 \mathrm{~Hz}, 1 \mathrm{H}, \mathrm{CH}$ ), $7.35(\mathrm{~d}, J=8.27 \mathrm{~Hz}, 1 \mathrm{H}, \mathrm{CH}), 4.43(\mathrm{t}, J=8.86 \mathrm{~Hz}, \mathrm{H}, \mathrm{CH})$, $3.52\left(\mathrm{dt}, J=7.21,1.52 \mathrm{~Hz}, 2 \mathrm{H}, \mathrm{CH}_{2}\right), 2.45\left(\mathrm{~s}, 3 \mathrm{H}, \mathrm{CH}_{3}\right), 2.41$ $\left(\mathrm{dd}, J=15.41,7.42 \mathrm{~Hz}, 2 \mathrm{H}, \mathrm{CH}_{2}\right), 2.08-2.13\left(\mathrm{~m}, 2 \mathrm{H}, \mathrm{CH}_{2}\right)$, $0.91\left(\mathrm{t}, J=7.36 \mathrm{~Hz}, 3 \mathrm{H}, \mathrm{CH}_{3}\right) ;{ }^{13} \mathrm{CNMR}(\mathrm{MeOD}, \delta / \mathrm{ppm})$ : 
172.1 (C-5), 168.3 (C-7), 145.3 (C-19), 135.4 (C-16), 125.9 (C-21), 63.5 (C-6), 44.1 (C-2), 30.8 (C-4), 25.3 (C-10), 17.4 (C-3), 11.9 (C-11); ESI-MS: $m / z 326.25[\mathrm{M}+2]^{+}, 324.27[\mathrm{M}]^{+}$.

2.4.3. 1-[3,4-Dihydroxy-5-(hydroxymethyl)tetrahydrofuran2-yl]-N-[(4-methylphenyl) sulfonyl]-1H-1,2, 4-triazole-3carboxamide (3c). $611.0 \mathrm{mg}(2.5 \mathrm{mmol})$ ribavirin reacts with $477.0 \mathrm{mg} p$-toluenesulfonyl chloride $(2.5 \mathrm{mmol})$ and $3 \mathrm{c}$ compound was obtained as white solid. IR $v_{\max }\left(\mathrm{cm}^{-1}\right)$ :

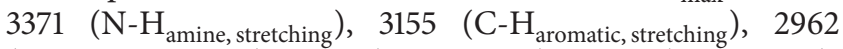
$\left(\mathrm{C}-\mathrm{H}_{\text {methyl, stretching }}\right), 1711\left(\mathrm{C}=\mathrm{O}_{\text {carbonyl }}\right), 1597\left(\mathrm{~N}-\mathrm{H}_{\text {bending }}\right)$, $1010\left(\mathrm{~S}=\mathrm{O}_{\text {stretching }}\right), 1174 \quad\left(-\mathrm{N}-\mathrm{S}=\mathrm{O}_{\text {stretching }}\right), 1496(\mathrm{C}=\mathrm{C}-$ $\left.\mathrm{C}_{\text {aromatic, stretching }}\right), 1357 \quad\left(\mathrm{O}-\mathrm{H}_{\text {bending }}\right), 678$ (Ø-S ${ }^{1} \mathrm{HNMR}$ (MeOD, $\left.\delta / \mathrm{ppm}\right): 8.88$ (s, $\left.1 \mathrm{H}, \mathrm{CH}\right), 7.81$ (d, $J=8.24 \mathrm{~Hz}, 1 \mathrm{H}, \mathrm{CH}), 7.45(\mathrm{~d}, J=8.27 \mathrm{~Hz}, 1 \mathrm{H}, \mathrm{CH})$, 7.03 (br s, H, NH), 5.92 (t, $J=5.31,1 \mathrm{H}, \mathrm{CH}), 4.45$ (t, $J=5.41$, $1 \mathrm{H}, \mathrm{CH}), 4.21(\mathrm{t}, J=5.31,1 \mathrm{H}, \mathrm{CH}), 4.12(\mathrm{t}, J=5.48,1 \mathrm{H}$, $\mathrm{CH}), 3.81\left(\mathrm{dd}, J=8.8,5.16 \mathrm{~Hz}, 2 \mathrm{H}, \mathrm{CH}_{2}\right), 2.43$ (s, $3 \mathrm{H}, \mathrm{CH}_{3}$ ); ${ }^{13} \mathrm{CNMR}$ (MeOD, $\left.\delta / \mathrm{ppm}\right): 157.1$ (C-8), 154.1 (C-15), 145.7 (C-24), 140.4 (C-10), 135.4 (C-21), 129.2 (C-23), 91.1 (C-2), 86.8 (C-5), 75.1 (C-3), 69.4 (C-4), 61.9 (C-3), 21 (C-27); ESI-MS: $m / z 400.35[\mathrm{M}+2]^{+}, 398.27[\mathrm{M}]^{+}$.

2.4.4. N-[(4-Methylphenyl) sulfonyl] Histidine (5a). Compound $5 \mathrm{a}$, as white solid, was obtained by the reaction of $518.0 \mathrm{mg}(3.34 \mathrm{mmol})$ histidine with $636.0 \mathrm{mg}$ (3.34 mmol) of $p$-toluenesulfonyl chloride. IR $v_{\max }\left(\mathrm{cm}^{-1}\right)$ : $3125\left(\mathrm{O}-\mathrm{H}_{\text {carboxylic, stretching }}\right), 2962\left(\mathrm{C}-\mathrm{H}_{\text {methyl, stretching }}\right), 1591$ ( $\left.\mathrm{N}-\mathrm{H}_{\text {bending }}\right), 1010\left(\mathrm{~S}=\mathrm{O}_{\text {stretching }}\right), 1170\left(-\mathrm{N}-\mathrm{S}=\mathrm{O}_{\text {stretching }}\right)$, $1496\left(\mathrm{C}=\mathrm{C}-\mathrm{C}_{\text {aromatic, stretching }}\right), 1650\left(\mathrm{C}=\mathrm{N}_{\text {stretching }}\right), 678$ (Ø$\left.\mathrm{S}_{\text {stretching }}\right) ;{ }^{1} \mathrm{HNMR}(\mathrm{MeOD}, \delta / \mathrm{ppm}): 8.31$ (s, $\left.1 \mathrm{H}, \mathrm{CH}\right), 7.91$ (s, $1 \mathrm{H}, \mathrm{CH}), 7.61(\mathrm{~d}, J=8.07 \mathrm{~Hz}, 1 \mathrm{H}, \mathrm{CH}), 7.33$ (br s, H, $\mathrm{NH}), 7.23(\mathrm{~d}, J=8.01,1 \mathrm{H}, \mathrm{CH}), 4.75(\mathrm{q}, J=8.21,1 \mathrm{H}$, $\mathrm{CH}$ ), 3.01 (qd, $\left.J=14.58,8.31,2 \mathrm{H}, \mathrm{CH}_{2}\right), 2.39$ (s, 3H, $\mathrm{CH}_{3}$ ); ${ }^{13}$ CNMR (MeOD, $\left.\delta / p p m\right): 175.1$ (C-11), 143.2 (C-17), 135.7 (C-2), 130.1 (C-16), 125.4 (C-15), 53.9 (C-7), 31.2 (C-6), 21.5 (C-21); ESI-MS: $m / z$ 311.28 [M+2] ${ }^{+}, 309.29[\mathrm{M}]^{+}$.

2.4.5. N-[(4-Methylphenyl) sulfonyl] Tryptophan (5b). Compound $5 \mathrm{~b}$, as white solid, was obtained by the reaction of $511.0 \mathrm{mg}(2.5 \mathrm{mmol})$ tryptophan with $477.0 \mathrm{mg}$ $(2.5 \mathrm{mmol})$ of $p$-toluenesulfonyl chloride. IR $v_{\max }\left(\mathrm{cm}^{-1}\right)$ : $3300\left(\mathrm{~N}-\mathrm{H}_{\text {amine, stretching }}\right), 3025\left(\mathrm{O}-\mathrm{H}_{\text {carboxylic, stretching }}\right), 1581$ (N$\left.\mathrm{H}_{\text {bending }}\right), 1040$ ( $\left.\mathrm{S}=\mathrm{O}_{\text {stretching }}\right), 1165\left(-\mathrm{N}-\mathrm{S}=\mathrm{O}_{\text {stretching }}\right), 1496$ $\left(\mathrm{C}=\mathrm{C}-\mathrm{C}_{\text {aromatic, stretching }}\right), 708\left(\varnothing-\mathrm{S}_{\text {stretching }}\right) ;{ }^{1} \mathrm{HNMR}(\mathrm{MeOD}$, $\delta / \mathrm{ppm}): 8.01$ (br s, $1 \mathrm{H}, \mathrm{NH}), 7.82(\mathrm{~d}, J=7.76 \mathrm{~Hz}, 1 \mathrm{H}, \mathrm{CH}), 7.71$ (s, H, CH), 7.65 (d, $J=8.07 \mathrm{~Hz}, 1 \mathrm{H}, \mathrm{CH}), 7.23(\mathrm{~d}, J=8.17 \mathrm{~Hz}$, $1 \mathrm{H}, \mathrm{CH}), 7.13(\mathrm{t}, J=8.11,1 \mathrm{H}, \mathrm{CH}), 6.75(\mathrm{~d}, J=7.71,1 \mathrm{H}$, $\mathrm{CH}), 4.61(\mathrm{t}, J=8.31,1 \mathrm{H}, \mathrm{CH}), 3.50(\mathrm{dt}, J=14.33,8.23,2 \mathrm{H}$, $\left.\mathrm{CH}_{2}\right), 2.39\left(\mathrm{~s}, 3 \mathrm{H}, \mathrm{CH}_{3}\right) ;{ }^{13} \mathrm{CNMR}(\mathrm{MeOD}, \delta / \mathrm{ppm}): 175.9(\mathrm{C}-$ 15), 143.5 (C-21), 134.7 (C-2), 130.1 (C-22), 122.9 (C-9), 118.6 (C-4), 111.8 (C-3), 63.5 (C-11), 29.7 (C-10), 21.4 (C-25); ESIMS: $m / z 360.45[\mathrm{M}+2]^{+}, 358.37[\mathrm{M}]^{+}$.

2.4.6. Methyl N-[(4-Methylphenyl) sulfonyl]- $\alpha$-aspartyl-Lphenylalaninate (7a). White crystals of compound 7 a were obtained by charging $589.0 \mathrm{mg}$ aspartame $(2.0 \mathrm{mmol})$ with
$381.0 \mathrm{mg}$ of $p$-toluenesulfonyl chloride $(2.0 \mathrm{mmol})$. IR $v_{\max }$ $\left(\mathrm{cm}^{-1}\right): 3340\left(\mathrm{~N}-\mathrm{H}_{\text {amine, stretching }}\right), 2950\left(\mathrm{O}-\mathrm{H}_{\text {carboxylic, stretching }}\right)$, $1219\left(\mathrm{C}-\mathrm{N}_{\text {amine, stretching }}\right), 1735\left(\mathrm{C}=\mathrm{O}_{\text {ester, stretching }}\right) 1581(\mathrm{~N}-$ $\left.\mathrm{H}_{\text {bending }}\right), 1040$ ( $\left.\mathrm{S}=\mathrm{O}_{\text {stretching }}\right), 1170\left(-\mathrm{N}-\mathrm{S}=\mathrm{O}_{\text {stretching }}\right), 1496$ $\left(\mathrm{C}=\mathrm{C}-\mathrm{C}_{\text {aromatic, stretching }}\right), 700$ (Ø-S stretching $) ;{ }^{1} \mathrm{HNMR}(\mathrm{MeOD}$, $\delta / \mathrm{ppm}): 7.61$ (br s, $1 \mathrm{H}, \mathrm{NH}), 7.42(\mathrm{~d}, J=8.06 \mathrm{~Hz}, 1 \mathrm{H}, \mathrm{CH}), 7.25$ $(\mathrm{d}, J=7.37 \mathrm{~Hz}, 1 \mathrm{H}, \mathrm{CH}), 6.93(\mathrm{~d}, J=8.14 \mathrm{~Hz}, 1 \mathrm{H}, \mathrm{CH}), 5.33$ $(\mathrm{d}, J=9.11,1 \mathrm{H}, \mathrm{CH}), 4.65(\mathrm{~d}, J=7.67,1 \mathrm{H}, \mathrm{CH}), 3.61(\mathrm{~s}, 3 \mathrm{H}$, $\left.\mathrm{CH}_{3}\right), 2.93\left(\mathrm{dt}, \mathrm{J}=13.73,6.83,2 \mathrm{H}, \mathrm{CH}_{2}\right), 2.39\left(\mathrm{~s}, 3 \mathrm{H}, \mathrm{CH}_{3}\right)$; ${ }^{13}$ CNMR (MeOD, $\left.\delta / p p m\right): 174.3$ (C-21), 170.7 (C-12), 143.5 (C-27), 136.8 (C-1), 129.7 (C-6), 126.1 (C-4), 55.6 (C-8), 52.3 (C-19), 37.8 (C-7), 21.5 (C-31); ESI-MS: $m / z 450.43[\mathrm{M}+2]^{+}$, $448.47[\mathrm{M}]^{+}$.

2.4.7. trans-4-(\{[(4-Methylphenyl)sulfonyl]amino $\}$ methyl $)$ cyclohexanecarboxylic Acid (9a). White crystals of compound 9a were obtained by charging $393.0 \mathrm{mg}$ tranexamic acid $(2.5 \mathrm{mmol})$ with $477.0 \mathrm{mg}$ of $p$-toluenesulfonyl chloride $(2.5 \mathrm{mmol})$. IR $v_{\max }\left(\mathrm{cm}^{-1}\right): 3263\left(\mathrm{~N}-\mathrm{H}_{\text {amine, stretching }}\right), 2962$ $\left(\mathrm{O}-\mathrm{H}_{\text {carboxylic, stretching }}\right), 1219\left(\mathrm{C}-\mathrm{N}_{\text {amine, stretching }}\right), 1597$ (N$\left.\mathrm{H}_{\text {bending }}\right), 1067$ ( $\left.\mathrm{S}=\mathrm{O}_{\text {stretching }}\right), 1157\left(-\mathrm{N}-\mathrm{S}=\mathrm{O}_{\text {stretching }}\right), 1496$ $\left(\mathrm{C}=\mathrm{C}-\mathrm{C}_{\text {aromatic, stretching }}\right), 680\left(\varnothing-\mathrm{S}_{\text {stretching }}\right) ;{ }^{1} \mathrm{HNMR}(\mathrm{MeOD}$, $\delta / \mathrm{ppm}): 7.71(\mathrm{~d}, J=8.06 \mathrm{~Hz}, 1 \mathrm{H}, \mathrm{CH}), 7.56$ (br s, H, NH), $7.22(\mathrm{~d}, J=8.06 \mathrm{~Hz}, 1 \mathrm{H}, \mathrm{CH}), 2.85(\mathrm{~d}, J=15.17 \mathrm{~Hz}, 1 \mathrm{H}$, $\mathrm{CH}), 2.33\left(\mathrm{~s}, 3 \mathrm{H}, \mathrm{CH}_{3}\right), 2.29(\mathrm{~d}, J=10.71,1 \mathrm{H}, \mathrm{CH}), 1.65(\mathrm{dt}$, $\left.J=11.67,9.71,2 \mathrm{H}, \mathrm{CH}_{2}\right), 1.41(\mathrm{qd}, J=12.93,10.83,2 \mathrm{H}$, $\left.\mathrm{CH}_{2}\right) ;{ }^{13} \mathrm{CNMR}$ (MeOD, $\left.\delta / \mathrm{ppm}\right): 182.5$ (C-11), 140.3 (C-17), 136.5 (C-14), 129.5 (C-16), 46.8 (C-7), 38.5 (C-1), 29.6 (C-6), 22.3 (C-21); ESI-MS: $m / z 313.35[\mathrm{M}+2]^{+}, 311.37[\mathrm{M}]^{+}$.

2.4.8. 4-Chloro-2-[(2-furylmethyl)amino]-5-(\{[(4-methylphenyl)sulfonyl]amino\}sulfonyl)benzoic Acid (11a). Compound 1la, as white solid, was obtained by the reaction of $661.0 \mathrm{mg}$ $(2.0 \mathrm{mmol})$ furosemide with $381.0 \mathrm{mg} \quad(2.0 \mathrm{mmol})$ of p-toluenesulfonyl chloride. IR $v_{\max }\left(\mathrm{cm}^{-1}\right): 3348(\mathrm{~N}-$ $\left.\mathrm{H}_{\text {amine, stretching }}\right), \quad 3250 \quad\left(\mathrm{O}-\mathrm{H}_{\text {carboxylic, stretching }}\right), 1607 \quad(\mathrm{~N}-$ $\left.\mathrm{H}_{\text {bending }}\right), 1072\left(\mathrm{~S}=\mathrm{O}_{\text {stretching }}\right), 1160\left(-\mathrm{N}-\mathrm{S}=\mathrm{O}_{\text {stretching }}\right), 1496$ $\left(\mathrm{C}=\mathrm{C}-\mathrm{C}_{\text {aromatic, stretching }}\right), 702\left(\varnothing-\mathrm{S}_{\text {stretching }}\right) ;{ }^{1} \mathrm{HNMR}$ (MeOD, $\delta / \mathrm{ppm}): 11.81$ (br s, H, NH), 8.42 (s, 1H, CH), 7.89 (d, $J=8.17 \mathrm{~Hz}, 1 \mathrm{H}, \mathrm{CH}), 7.58(\mathrm{~d}, J=8.11 \mathrm{~Hz}, 1 \mathrm{H}, \mathrm{CH}), 7.39(\mathrm{~s}$, $\mathrm{H}, \mathrm{CH}), 6.65$ (s, H, CH), 6.21 (s, H, CH), 6.16 (d, $J=3.27$, $\mathrm{H}, \mathrm{CH}), 4.41$ (s, H, CH), 2.42 (s, H, CH); ${ }^{13} \mathrm{CNMR}$ (MeOD,

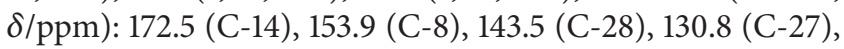
123.8 (C-13), 120.5 (C-9), 110.4 (C-4), 42.3 (C-6), 21.5 (C-31); ESI-MS: $m / z 486.95[\mathrm{M}+2]^{+}, 484.87[\mathrm{M}]^{+}$.

2.4.9. 4-Methyl-N-(\{4-[5-(4-methylphenyl)-3-(trifluoromethyl)-1H-pyrazol-1-yl]phenyl\}sulfonyl)Benzenesulfonamide(11b). Compound 11b, as white solid, was obtained by the reaction of $424.0 \mathrm{mg}(1.11 \mathrm{mmol})$ celecoxib with $212.0 \mathrm{mg}(1.11 \mathrm{mmol})$ of $p$-toluenesulfonyl chloride. IR $v_{\max }\left(\mathrm{cm}^{-1}\right): 3327(\mathrm{~N}-$ $\left.\mathrm{H}_{\text {amine, stretching }}\right), 1607$ ( $\left.\mathrm{N}-\mathrm{H}_{\text {bending }}\right), 1065$ ( $\left.\mathrm{S}=\mathrm{O}_{\text {stretching }}\right)$, $1142\left(-\mathrm{N}-\mathrm{S}=\mathrm{O}_{\text {stretching }}\right), 1502\left(\mathrm{C}=\mathrm{C}-\mathrm{C}_{\text {aromatic, stretching }}\right), 1650$ $\left(\mathrm{C}=\mathrm{N}_{\text {stretching }}\right), 703$ (Ø- $\left.\mathrm{S}_{\text {stretching }}\right), 1095-1125\left(\mathrm{C}-\mathrm{F}_{\text {stretching }}\right)$; ${ }^{1} \mathrm{HNMR}$ (MeOD, $\delta / \mathrm{ppm}$ ): 16.11 (br s, H, NH), 8.62 (d, $J=8.19 \mathrm{~Hz}, 1 \mathrm{H}, \mathrm{CH}), 8.37(\mathrm{~d}, J=8.01 \mathrm{~Hz}, 1 \mathrm{H}, \mathrm{CH}), 8.08$ (d, $J=9.91 \mathrm{~Hz}, 1 \mathrm{H}, \mathrm{CH}), 7.91(\mathrm{~d}, J=8.17 \mathrm{~Hz}, 1 \mathrm{H}, \mathrm{CH}), 7.65$ 

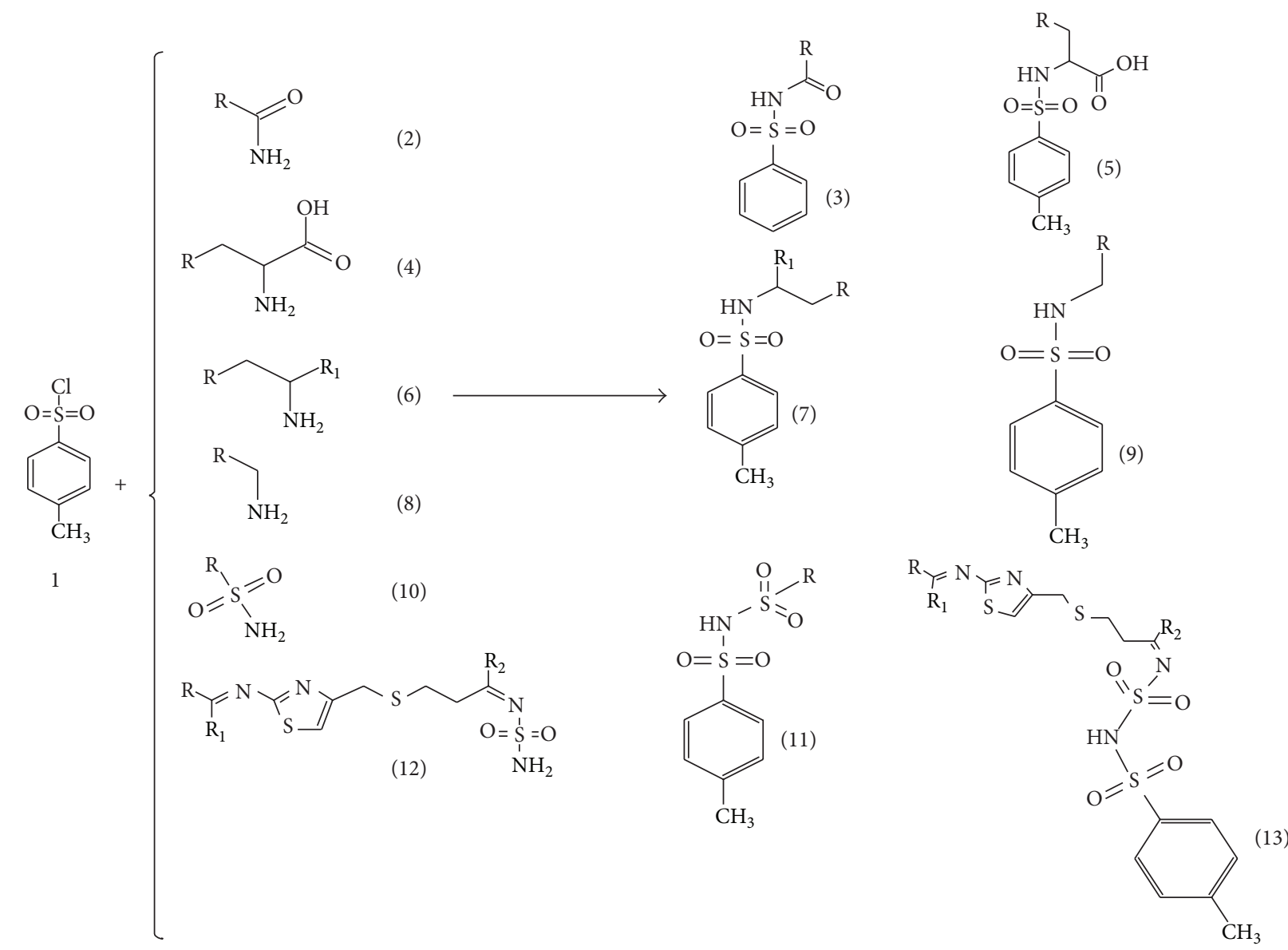

(3)

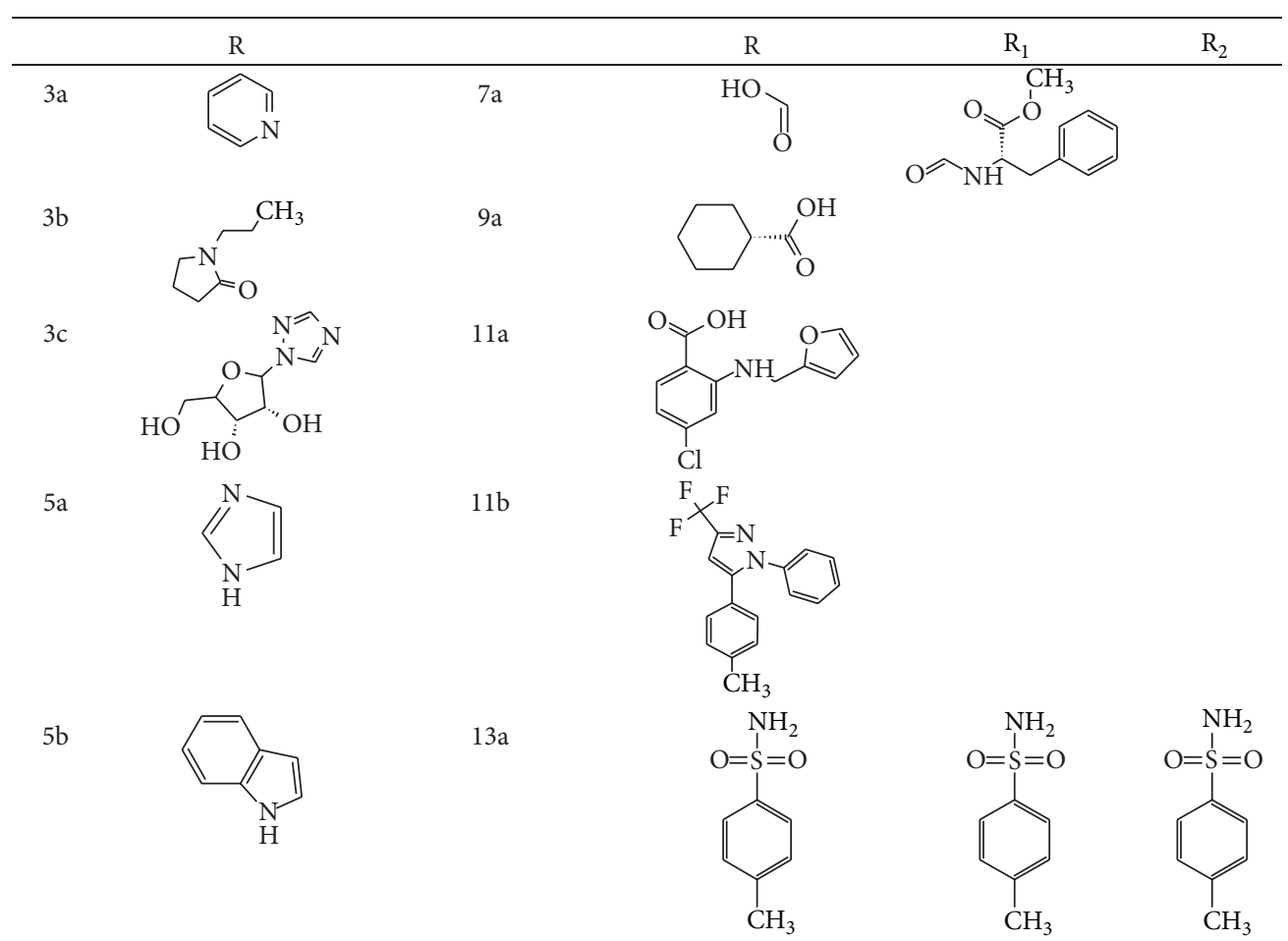

Scheme 1: Synthesis of sulfonamides. 


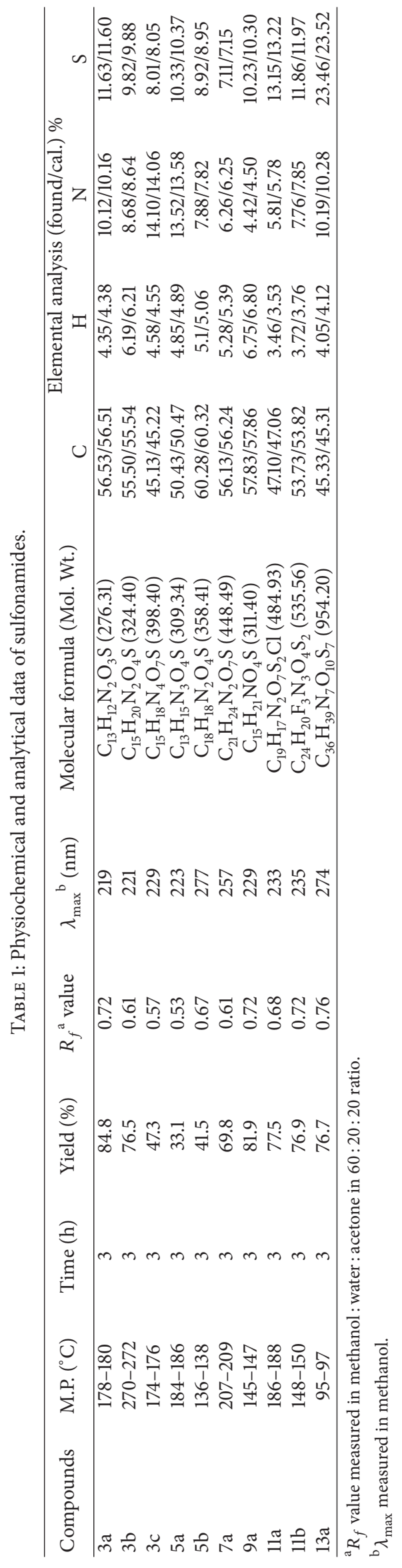


TABLE 2: Zone $\mathrm{a}^{\mathrm{a}}$ of inhibition and $\mathrm{MIC}^{\mathrm{b}}$ of sulfonamides against pathogenic bacterial strains.

\begin{tabular}{|c|c|c|c|c|c|c|c|c|}
\hline \multirow{4}{*}{ Compounds } & \multicolumn{8}{|c|}{ Name of bacteria } \\
\hline & \multicolumn{4}{|c|}{ Gram (+) bacterial strains } & \multicolumn{4}{|c|}{ Gram (-) bacterial strains } \\
\hline & \multicolumn{2}{|c|}{ S. aureus (ATCC 25923) } & \multicolumn{2}{|c|}{ B. subtilis (ATCC 6633) } & \multicolumn{2}{|c|}{ E. coli (ATCC 25922) } & \multicolumn{2}{|c|}{ K. pneumoniae (ATCC 13887) } \\
\hline & Zone of inhibition & MIC & Zone of inhibition & MIC & Zone of inhibition & MIC & Zone of inhibition & MIC \\
\hline $3 a$ & - & $>500$ & - & $>500$ & - & $>500$ & $26 \pm 1.33$ & 62.5 \\
\hline $3 b$ & $19 \pm 1.22$ & 125 & - & $>500$ & $28 \pm 0.91$ & 15.63 & $28 \pm 0.33$ & 62.5 \\
\hline $3 c$ & - & $>500$ & - & $>500$ & $18 \pm 1.32$ & 125 & - & $>500$ \\
\hline $5 \mathrm{a}$ & - & $>500$ & - & $>500$ & $31 \pm 0.12$ & 7.81 & $18 \pm 0.38$ & 125 \\
\hline $5 b$ & $19 \pm 0.81$ & 125 & - & $>500$ & $18 \pm 0.48$ & 125 & - & $>500$ \\
\hline $7 \mathrm{a}$ & - & $>500$ & - & $>500$ & - & $>500$ & - & $>500$ \\
\hline $9 a$ & - & $>500$ & - & $>500$ & $30 \pm 0.12$ & 7.81 & - & $>500$ \\
\hline $11 \mathrm{a}$ & $12 \pm 0.92$ & 250 & - & $>500$ & $18 \pm 1.51$ & 250 & $16 \pm 0.55$ & 250 \\
\hline $11 b$ & $18 \pm 1.04$ & 62.5 & - & $>500$ & $20 \pm 1.90$ & 250 & $18 \pm 0.87$ & 250 \\
\hline $13 a$ & - & $>500$ & - & $>500$ & $28 \pm 0.53$ & 125 & $18 \pm 0.91$ & 125 \\
\hline Ciprofloxacin $^{\mathrm{c}}$ & $32 \pm 0.22$ & 1.25 & $32 \pm 0.13$ & 1.25 & $32 \pm 0.12$ & 0.625 & $30 \pm 0.12$ & 0.625 \\
\hline
\end{tabular}

${ }^{\mathrm{a}}$ Zone of inhibition was measured in $\mathrm{mm}$.

${ }^{\mathrm{b}} \mathrm{MIC}$ (minimum inhibitory concentrations) were measured in $\mu \mathrm{g} / \mathrm{mL}$.

${ }^{\mathrm{c}}$ Control drug.

(d, $J=7.97 \mathrm{~Hz}, 1 \mathrm{H}, \mathrm{CH}), 6.81(\mathrm{~s}, \mathrm{H}, \mathrm{CH}), 2.46$ (s, H, CH), 2.35 (s, H, CH); ${ }^{13} \mathrm{CNMR}$ (MeOD, $\left.\delta / \mathrm{ppm}\right): 147.2(\mathrm{C}-5), 141.8$ (C-33), 138.5 (C-13), 131.8 (C-9), 128.3 (C-31), 117.5 (C-12), 110.7 (C-4), 21.5 (C-24); ESI-MS: $m / z 537.55[\mathrm{M}+2]^{+}, 535.57$ $[\mathrm{M}]^{+}$.

2.4.10. (1E)-3-[(\{2-[(Bis $\{[(4-m e t h y l p h e n y l)$ sulfonyl] amino $\}$ methylene) amino]-1,3-thiazol-4-yl\}methyl) thio]-N-[(4methylphenyl) sulfonyl $]-N^{\prime}-(\{[(4-m e t h y l p h e n y l)$ sulfonyl $] a m-$ ino\} sulfonyl) Propanimidamide (13a). Compound 13a, as white solid, was obtained by the reaction of $282.0 \mathrm{mg}$ famotidine $(0.833 \mathrm{mmol})$ with $635.0 \mathrm{mg}$ of $p$-toluenesulfonyl chloride $\quad(3.34 \mathrm{mmol})$. IR $v_{\max }\left(\mathrm{cm}^{-1}\right): 3327 \quad(\mathrm{~N}-$

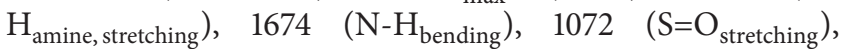
$1172\left(-\mathrm{N}-\mathrm{S}=\mathrm{O}_{\text {stretching }}\right), 1496\left(\mathrm{C}=\mathrm{C}-\mathrm{C}_{\text {aromatic, stretching }}\right), 1650$ $\left(\mathrm{C}=\mathrm{N}_{\text {stretching }}\right), 702\left(\varnothing-\mathrm{S}_{\text {stretching }}\right), 652\left(\mathrm{C}-\mathrm{S}_{\text {stretching }}\right) ;{ }^{1} \mathrm{HNMR}$ (MeOD, $\delta / \mathrm{ppm}): 9.51$ (br s, H, NH), $8.12(\mathrm{~d}, J=8.19 \mathrm{~Hz}$, $1 \mathrm{H}, \mathrm{CH}), 7.87(\mathrm{~d}, J=8.11 \mathrm{~Hz}, 1 \mathrm{H}, \mathrm{CH}), 7.68(\mathrm{~d}, J=8.91 \mathrm{~Hz}$, $1 \mathrm{H}, \mathrm{CH}), 7.31(\mathrm{~d}, J=8.10 \mathrm{~Hz}, 1 \mathrm{H}, \mathrm{CH}), 3.85$ (s, H, CH), $2.93(\mathrm{~d}, J=15.97 \mathrm{~Hz}, 1 \mathrm{H}, \mathrm{CH}), 2.81\left(\mathrm{~s}, 2 \mathrm{H}, \mathrm{CH}_{2}\right), 2.66(\mathrm{~s}$, $\left.3 \mathrm{H}, \mathrm{CH}_{3}\right), 2.36\left(\mathrm{~s}, 3 \mathrm{H}, \mathrm{CH}_{3}\right), 2.30\left(\mathrm{~s}, 3 \mathrm{H}, \mathrm{CH}_{3}\right) ;{ }^{13} \mathrm{CNMR}$ (MeOD, $\delta / \mathrm{ppm}): 174.8$ (C-15), 164.1 (C-2), 153.5 (C-4), 144.4 (C-7), 132.1 (C-54), 126.5 (C-38), 104.5 (C-5), 35.2 (C-11), 32.3 (C-13), 21.2 (C-59); ESI-MS: $m / z$ 956.21 [M+2] ${ }^{+}, 954.17$ $[\mathrm{M}]^{+}$.

\section{Results and Discussion}

A series of ten sulfonamides were synthesized in aqueous basic media by simple reaction of six amino group containing drugs; two amino acids and two amino acid analogs (nicotinamide is used as source of vitamin B and aspartame is sweetener used in various pharmaceutical liquid formulations) with paratoluene sulphonyl chloride with continuous stirring and details of reaction conditions are explained in experimental section and synthetic pathway of sulfonamides is explained in Scheme 1. The compounds 3a and 9a were obtained in excellent yield (above $80 \%$ ) while the 3c, 5a, and $5 \mathrm{~b}$ gave the poor yield (below 50\%). The remaining compounds were obtained in good yield (69-77\%). Elemental analysis was performed for the conformation of all the compounds and measurement of absorption maximum $\left(\lambda_{\max }\right)$ provided the justification. The physiochemical and analytical data of synthesized sulfonamides are presented in Table 1 . The synthesized compounds were characterized by FT-IR; the characteristics band at $3263-3371 \mathrm{~cm}^{-1}$ of $\mathrm{N}-\mathrm{H}$ amide stretching and $1174-1127 \mathrm{~cm}^{-1}$ for $(-\mathrm{N}-\mathrm{S}=\mathrm{O})$ and $1072-$ $1010 \mathrm{~cm}^{-1}(\mathrm{~S}=\mathrm{O})$ for all compounds reveals the formation of sulfonamides. $[\mathrm{M}+2]^{+}$peaks obtained by ESI-MS represented the isolation of sulfonyl group in all synthesized compounds. The structures of all the compounds were also confirmed by ${ }^{1} \mathrm{HNMR}$ and ${ }^{13} \mathrm{CNMR}$ by dissolving in MeOD. ${ }^{1} \mathrm{HNMR}$ spectra of compounds $3 \mathrm{c}, 5 \mathrm{a}, 7 \mathrm{a}$, and $9 \mathrm{a}$ showed a signal at $\delta$ 7.03-7.61, while a signal at $\delta 16.11$ and $11.81 \mathrm{ppm}$ for $11 \mathrm{~b}$ and $11 \mathrm{a}$ corresponds to $\mathrm{NH}$ group of sulfonamide.

A broad singlet due to $-\mathrm{NH}$ group was also obtained for compounds $3 \mathrm{a}, 3 \mathrm{~b}, 5 \mathrm{~b}$, and $13 \mathrm{a}$ at $\delta 8.38,9.68,8.01$, and $9.51 \mathrm{ppm}$, respectively. The characteristics C- SO-NH signals at $\delta 131-139 \mathrm{ppm}$ of all the compounds were shown by ${ }^{13} \mathrm{CNMR}$ which identified the structures correctly. Synthesized compounds were also screened for their antibacterial activities against gram negative bacterial $E$. coli and $K$. pneumoniae and gram positive $S$. aureus and B. subtilis by following the guidelines of CLSI $[12,13]$ using ciprofloxacin as reference antibacterial agent. Among the bacterial strains, the compounds $3 \mathrm{a}$ and $3 \mathrm{~b}$ have excellent antibacterial activities against K. pneumoniae with zone of inhibition comparable with control drug (MIC 62.5). Compounds 3c, 5b, 7a, and 9 a showed moderate activities while remaining compounds have no activity against the prescribed bacterial strain. Compounds 5a and 9a exhibited excellent activities against 
E. coli almost the same zone of inhibition as by reference ciprofloxacin (MIC 7.81), while $3 a$ and 7a showed no activity $(\mathrm{MIC}>500)$. The remaining compounds were moderate active against the mentioned strain. All the compounds were totally inactive against the B. subtilis. Compounds $3 \mathrm{~b}$ (MIC 125), 5b (MIC 125), and 11b (MIC 62.5) showed moderate activity, while 1la exhibited poor activity against the $S$. aureus and remaining compounds are totally inactive. The MIC values and zone of inhibitions are presented in Table 2 .

\section{Conclusion}

In conclusion, ten novel sulfonamides were synthesized; the reactions conditions are easy and excellent yields of compounds were obtained and progress of reaction was monitored by TLC and their structures were confirmed by spectral and elemental analysis. All the synthesized compounds were evaluated for their antibacterial activities and the results of their bioassay indicated that the sulfonamides attached to amino acid (histidine) and antifibrinolytic (tranexamic acid) showed antibacterial activities comparable to ciprofloxacin although these two agents alone have no antibacterial activity. The results confirmed that the compounds which are inactive against bacterial strains showed antibacterial activities after formation of sulfonamides.

\section{Conflict of Interests}

The authors declare that there is no conflict of interests regarding the publication of this paper.

\section{References}

[1] A. Ali, G. S. K. K. Reddy, H. Cao et al., "Discovery of HIV1 protease inhibitors with picomolar affinities incorporating $\mathrm{N}$-aryl oxazolidinone-5-carboxamides as novel p2 ligands," Journal of Medicinal Chemistry, vol. 49, no. 25, pp. 7342-7356, 2006.

[2] R. Li, G. L. Kenyon, F. E. Cohen et al., "In vitro antimalarial activity of chalcones and their derivatives," Journal of Medicinal Chemistry, vol. 38, no. 26, pp. 5031-5037, 1995.

[3] B. F. Abdel-Wahab, H. A. Abdel-Aziz, and E. M. Ahmed, "Synthesis and antimicrobial evaluation of some 1,3-thiazole, 1,3,4-thiadiazole, 1,2,4-triazole, and 1,2,4-triazolo[3,4-b][1,3,4]thiadiazine derivatives including a 5-(benzofuran-2-yl)-1phenylpyrazole moiety," Monatshefte fur Chemie, vol. 140, no. 6, pp. 601-605, 2009.

[4] B. Siesky, A. Harris, E. Brizendine et al., "Literature review and meta-analysis of topical carbonic anhydrase inhibitors and ocular blood flow," Survey of Ophthalmology, vol. 54, no. 1, pp. 33-46, 2009.

[5] F. A. N. El-Dien, G. G. Mohamed, E. Khaled, and E. Y. Z. Frag, "Extractive spectrophotometric determination of sulphonamide drugs in pure and pharmaceutical preparations through ion-pair formation with molybdenum(V) thiocyanate in acidic medium," Journal of Advanced Research, vol. 1, no. 3, pp. 215-220, 2010.

[6] L. Saíz-Urra, M. P. González, I. G. Collado, and R. Hernández-Galán, "Quantitative structure-activity relationship studies for the prediction of antifungal activity of $\mathrm{N}$ arylbenzenesulfonamides against Botrytis cinerea," Journal of Molecular Graphics and Modelling, vol. 25, no. 5, pp. 680-690, 2007.

[7] W.-J. Zhu, P. Wu, X.-M. Liang et al., "Design, synthesis, and fungicidal activity of macrolactones and macrolactams with a sulfonamide side chain," Journal of Agricultural and Food Chemistry, vol. 56, no. 15, pp. 6547-6553, 2008.

[8] I. R. Ezabadi, C. Camoutsis, P. Zoumpoulakis et al., "Sulfonamide-1,2,4-triazole derivatives as antifungal and antibacterial agents: synthesis, biological evaluation, lipophilicity, and conformational studies," Bioorganic \& Medicinal Chemistry, vol. 16, no. 3, pp. 1150-1161, 2008.

[9] M. A. Gonzalez, D. B. Gorman, C. T. Hamilton, and G. A. Roth, "Process development for the sulfonamide herbicide pyroxsulam," Organic Process Research \& Development, vol. 12, no. 2, pp. 301-303, 2008.

[10] G. H. Jeffery, J. Bassett, J. Mendham, and R. C. Denney, Vogel's Textbook of Quantitative Chemical Analysis, John Wiley \& Sons, New York, NY, USA, 1989.

[11] X. Deng and N. S. Mani, "A facile, environmentally benign sulfonamide synthesis in water," Green Chemistry, vol. 8, no. 9, pp. 835-838, 2006.

[12] Clinical and Laboratory Standard Institute, Performance Standard for Anti-Microbial Disk Susceptibility Tests: Approved Standard M2-A-9, Clinical and Laboratory Standard Institute, Wayne, Pa, USA, 2006.

[13] Clinical and Laboratory Standard Institute, Method for Dilution Anti-Microbial Susceptibility Tests for Bacteria That Grow Aerobically; Approved Standard M7-a-9, Clinical and Laboratory Standard Institute, Wayne, Pa, USA, 2006. 

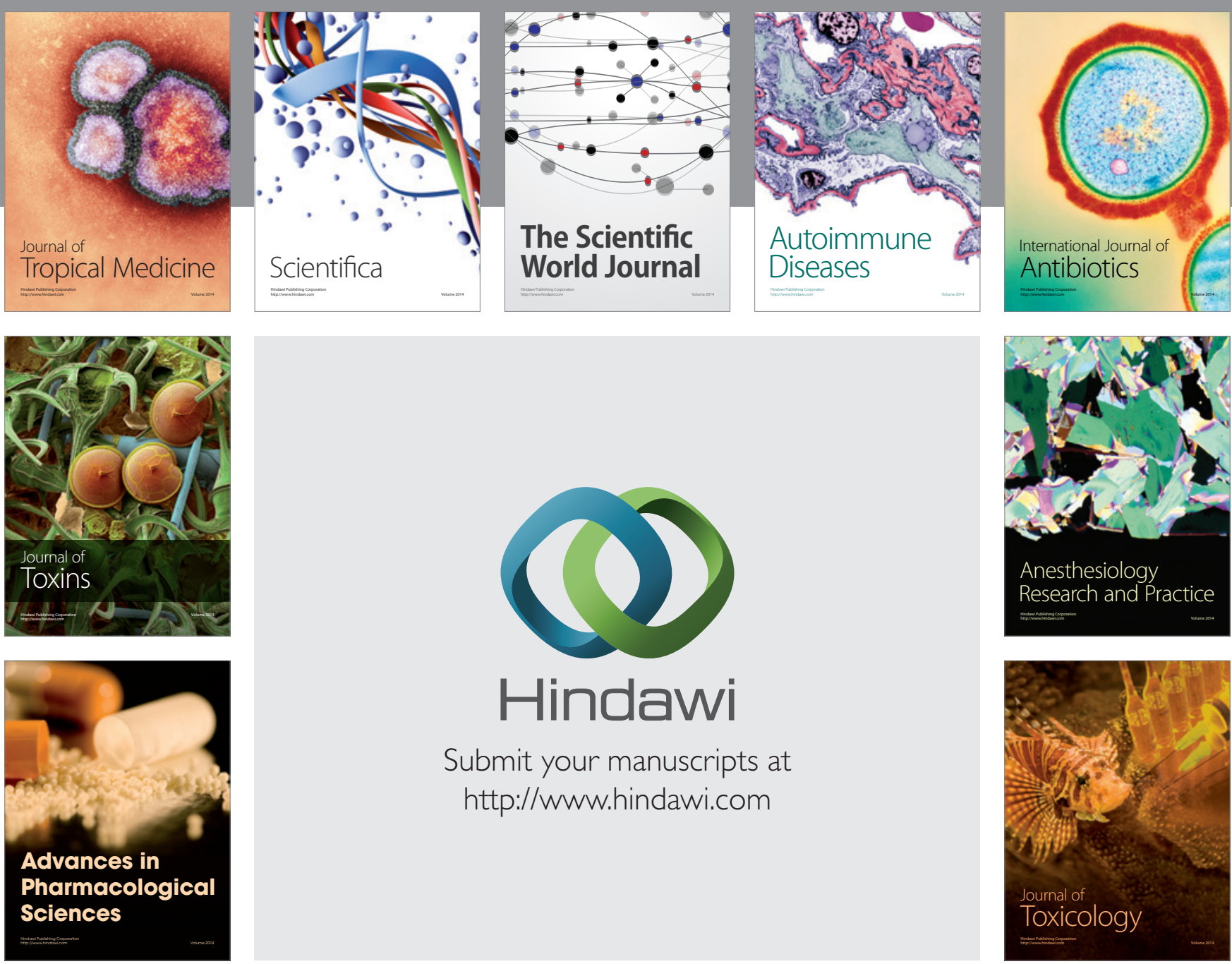

\section{Hindawi}

Submit your manuscripts at

http://www.hindawi.com
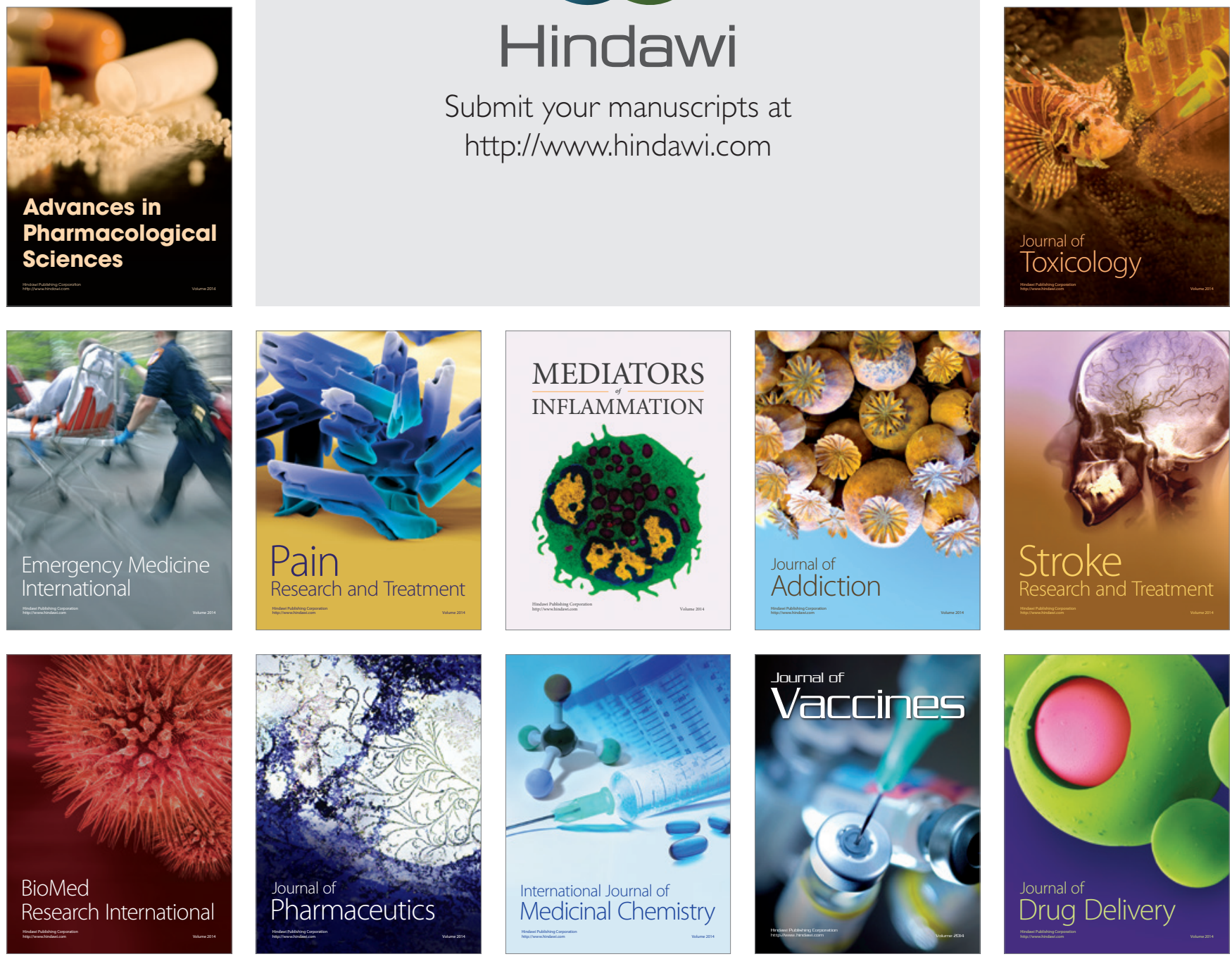